\title{
FEEDBACK STABILIZATION OF THE 3-D NAVIER- STOKES EQUATIONS BASED ON AN EXTENDED SYSTEM
}

\author{
M. Badra ${ }^{1}$ \\ ${ }^{1}$ Laboratoire MIP, UMR CNRS 5640, Université Paul Sabatier, 31062 Toulouse Cedex 4, France, \\ badra@mip.ups-tlse.fr
}

Abstract We study the local exponential stabilization of the 3D Navier-Stokes equations in a bounded domain, around a given steady-state flow, by means of a boundary control. We look for a control so that the solution to the Navier-Stokes equation be a strong solution. In the 3D case, such solutions may exist if the Dirichlet control satisfies a compatibility condition with the initial condition. In order to determine a feedback law satisfying such a compatibility condition, we consider an extended system coupling the Navier-Stokes equations with an equation satisfied by the control on the boundary of the domain. We determine a linear feedback law by solving a linear quadratic control problem for the linearized extended system. We show that this feedback law also stabilizes the nonlinear extended system. tion.

Keywords: Navier-Stokes equation, Feedback stabilization, Riccati equa-

\section{Introduction}

Let $\mathcal{O}$ and $\mathcal{B}$ two regular bounded domains of class $C^{\infty}$ in $\mathbf{R}^{3}$ such that $\overline{\mathcal{B}} \subset \mathcal{O}, \Omega=\mathcal{O} \backslash \overline{\mathcal{B}}, \Gamma_{e}=\partial \mathcal{O}$ and $\Gamma_{i}=\partial \mathcal{B}$. We have $\Gamma_{i} \cap \Gamma_{e}=\emptyset$ and $\partial \Omega=\Gamma_{i} \cup \Gamma_{e}$. We consider the motion of an incompressible fluid around the bounded body $\mathcal{B}$ in $\Omega$ which is described by the couple $\left(z_{e}, p_{e}\right)$, the velocity and the pressure, solution to the stationary Navier-Stokes equations

$$
\begin{aligned}
& -\Delta z_{e}+\left(z_{e} \cdot \nabla\right) z_{e}+\nabla p_{e}=0 \text { and } \nabla \cdot z_{e}=0 \text { in } \Omega \\
& z_{e}=0 \text { on } \Gamma_{i}, \quad z_{e}=v_{\infty} \text { on } \Gamma_{e} .
\end{aligned}
$$

According to [4], if $v_{\infty} \in H^{\frac{3}{2}}\left(\Gamma_{i} ; \mathbf{R}^{3}\right)$ obeys $\int_{\Gamma_{e}} v_{\infty} \cdot n=0$, such a stationary solution exists in $H^{2}\left(\Omega, \mathbf{R}^{3}\right) \times H^{1}(\Omega) / \mathbf{R}$. For an initial condition of the form $z_{e}+z_{0}$ and a Dirichlet boundary control $u$ on $\Gamma_{i}$ such that $\int_{\Gamma_{i}} u(t) \cdot n=0$, the pair $\left(z+z_{e}, p+p_{e}\right)$ satisfies the instationary Navier-Stokes equations, and

Please use the following format when citing this chapter:

Badra, M., 2006, in IFIP International Federation for Information Processing, Volume 202, Systems, Control, Modeling and Optimization, eds. Ceragioli, F., Dontchev, A., Furuta, H., Marti, K., Pandolfi, L., (Boston: Springer), pp. 13-24. 
$(z, p)$ obeys:

$$
\begin{aligned}
& \partial_{t} z-\Delta z+(z \cdot \nabla) z_{e}+\left(z_{e} \cdot \nabla\right) z+(z \cdot \nabla) z+\nabla p=0 \text { in } Q \\
& \nabla \cdot z=0 \text { in } Q, z=u \text { on } \Sigma_{i}, z=0 \text { on } \Sigma_{e}, z(0)=z_{0} .
\end{aligned}
$$

In this setting $Q=\Omega \times(0, \infty), \Sigma_{i}=\Gamma_{i} \times(0, \infty), \Sigma_{e}=\Gamma_{e} \times(0, \infty)$ and $n$ denotes the unit normal vector to $\Gamma_{i}$, exterior to $\Omega$. We assume that $z_{e}$ is an unstable solution of (1)-(2) corresponding to $z_{0}=z_{e}$. Our goal is to find a Dirichlet boundary control $u$ on $\Gamma_{i}$ which stabilizes the instationary Navier-Stokes system (1)-(2) for initial data $z_{0}$ small enough in an appropriate functional space. To achieve this goal, the three dimensional case is hightly demanding in terms of velocity regularity: we need that $z \in L^{2}\left(0, \infty ; \mathbf{H}^{\frac{3}{2}}(\Omega)\right)$ to obtain a stabilization result. Therefore, we look for a control $u$ regular enough to fit the expected smoothness of $z$ and in particular, the initial compatibility condition $u(0)=\left.z_{0}\right|_{\Gamma_{i}}$ should be satisfied. A way to obtain such compatibility condition is to characterize the trace $u$ as the first component of $(u, \sigma) \in L_{l o c}^{2}\left([0, \infty) ; L^{2}\left(\Gamma_{i} ; \mathbf{R}^{3}\right)\right) \times L_{l o c}^{2}([0, \infty))$, where $(u, \sigma)$ is the solution to the time dependent equation:

$$
\begin{aligned}
& \partial_{t} u=\Delta_{b} u+\sigma n+g \quad \text { on } \Sigma_{i}, \\
& u(0)=z_{0 \mid \Gamma_{i}}, \quad \text { and } \int_{\Gamma_{i}} u(t) \cdot n=0 .
\end{aligned}
$$

Here $\Delta_{b}$ is a Laplace Beltrami operator and $g \in L^{2}\left(\Sigma_{i} ; \mathbf{R}^{3}\right)$ is such that $g(t)$ obeys $\int_{\Gamma_{i}} g(t) \cdot n=0$. Thus the state $(z, u)$ now satisfy an extended system of two coupled equations with a distributed control $g$ on $\Gamma_{i}$ :

$$
\begin{aligned}
& \partial_{t} z-\Delta z+(z \cdot \nabla) z_{e}+\left(z_{e} \cdot \nabla\right) z+(z \cdot \nabla) z+\nabla p=0 \text { in } Q \\
& \nabla \cdot z=0 \text { in } Q, \quad z=u \text { on } \Sigma_{i}, \quad z=0 \text { on } \Sigma_{e}, \quad z(0)=z_{0} \\
& \partial_{t} u-\Delta_{b} u-\sigma n=g \text { in } \Sigma_{i}, \quad \int_{\Gamma_{i}} u(t) \cdot n=0, \quad u(0)=\left.z_{0}\right|_{\Gamma_{i}}
\end{aligned}
$$

In a first step, we consider the linear problem derived from this last coupled system by dropping the nonlinear term $(z \cdot \nabla) z$. We introduce the velocity space $V_{n}^{0}(\Omega)=\left\{y \in L^{2}\left(\Omega ; \mathbf{R}^{3}\right) \mid \nabla \cdot y=0\right.$ in $\Omega, y \cdot n=0$ on $\left.\partial \Omega\right\}$, and the orthogonal projector $P$ from $L^{2}\left(\Omega ; \mathbf{R}^{3}\right)$ into $V_{n}^{0}(\Omega)$. Next we rewrite the extended system as an evolution equation (see section 2.2 ) involving a linear unbounded operator $\mathcal{A}$ which is studied in section 4 . Then we state a linear quadratic optimal control problem (see section 2.3 ) which provides a distributed feedback controller for the extended system (see section 5). Finally, we apply the feedback controller to the initial nonlinear system (see section 6) and we show a local stabilization result. 


\section{Extended system and optimal control problem}

\subsection{Functional framework}

Let us define the spaces of free divergence functions

$$
\begin{aligned}
& V^{s}(\Omega)=\left\{y \in H^{s}\left(\Omega ; \mathbf{R}^{3}\right) \mid \nabla \cdot y=0 \text { in } \Omega, \int_{\partial \Omega} y \cdot n=0\right\}, \quad s \geq 0 \\
& V_{n}^{s}(\Omega)=\left\{y \in H^{s}\left(\Omega ; \mathbf{R}^{3}\right) \mid \nabla \cdot y=0 \text { in } \Omega, y \cdot n=0 \text { on } \partial \Omega\right\}, \quad s \geq 0
\end{aligned}
$$

and the corresponding trace spaces with a free mean normal component

$$
V^{s}\left(\Gamma_{i}\right)=\left\{y \in H^{s}\left(\Gamma_{i} ; \mathbf{R}^{3}\right) \mid \int_{\Gamma_{i}} y \cdot n=0\right\}, \quad V^{-s}\left(\Gamma_{i}\right)=V^{s}\left(\Gamma_{i}\right)^{\prime}, \quad s \geq 0 .
$$

We denote by $V_{0}^{s}(\Omega)$ the interpolation space $\left[V^{2}(\Omega) \cap H_{0}^{1}\left(\Omega ; \mathbf{R}^{3}\right), V_{n}^{0}(\Omega)\right]_{1-s / 2}$ for $0 \leq s \leq 2$ and $V^{-s}(\Omega)=V_{0}^{s}(\Omega)^{\prime}$ its dual counterpart with respect to the pivot space $V_{n}^{0}(\Omega)$. It is well known that

$$
\begin{aligned}
& V_{0}^{s}(\Omega)=V_{n}^{s}(\Omega), \quad 0 \leq s<\frac{1}{2} \\
& V_{0}^{\frac{1}{2}}(\Omega)=\left\{\left.y \in V_{n}^{\frac{1}{2}}(\Omega)\left|\int_{\Omega} \rho(x)^{-1}\right| y\right|^{2}<+\infty\right\} \\
& V_{0}^{s}(\Omega)=\left\{y \in V_{n}^{s}(\Omega) \mid y=0 \text { on } \partial \Omega\right\}, \quad \frac{1}{2}<s \leq 2
\end{aligned}
$$

where $\rho(x)$ is the distance from $x$ to $\partial \Omega$. Notice that, according to the above definition, we have $V_{0}^{s}(\Omega)=V^{s}(\Omega) \cap H_{0}^{1}\left(\Omega ; \mathbf{R}^{3}\right)$ for $1 \leq s \leq 2$. Finally, for $0<T \leq \infty$, and $X_{1}$ and $X_{2}$ two Banach spaces, we introduce the function space

$$
W\left(0, T ; X_{1}, X_{2}\right)=L^{2}\left(0, T ; X_{1}\right) \cap H^{1}\left(0, T ; X_{2}\right)
$$

\subsection{Abstract formulation of the extended system}

In this section, we state an abstract weak formulation for the system

$$
\begin{aligned}
& \partial_{t} z-\Delta z+(z \cdot \nabla) z_{e}+\left(z_{e} \cdot \nabla\right) z+\kappa(z \cdot \nabla) z+\nabla p=0 \text { in } Q \\
& \nabla \cdot z=0 \text { in } Q, z=u \text { on } \Sigma_{i}, z=0 \text { on } \Sigma_{e}, z(0)=z_{0} \in V^{0}(\Omega) \\
& \partial_{t} u-\Delta_{b} u-\sigma n=g \text { in } \Sigma_{i}, \quad u(0)=u_{0} \in V^{-\frac{1}{2}}\left(\Gamma_{i}\right)
\end{aligned}
$$

Equation (6) corresponds to the Oseen equation if $\kappa=0$, and to the NavierStokes equation if $\kappa=1$. Equation (6), the left hand side of (8) and (7) are satisfied in the sense of distributions. We observe that $\sigma$ plays the role of the Lagrange multiplier associated with the constraint $\int_{\Gamma_{i}} u \cdot n$.

By using transformations developed in [6] we are going to give an equivalent formulation of (6)-(7)-(8). First we define the unbounded operator $(\mathcal{D}(A), A)=$ $\left(V_{0}^{2}(\Omega), A(x, \partial)\right)$ where $A(x, \partial)=P \Delta-P\left(\nabla z_{e}\right)-P\left(z_{e} \cdot \nabla\right)$. We choose $\lambda_{0}>0$ such that $\left\langle\left(\lambda_{0}-A\right) y \mid y\right\rangle \geq \frac{1}{2}\|y\|_{V_{0}^{1}(\Omega)}$ for all $y \in V_{0}^{1}(\Omega)$, and we 
introduce the Dirichlet operator $D \in \mathcal{L}\left(V^{0}\left(\Gamma_{i}\right), V^{0}(\Omega)\right)$ associated with $\lambda_{0}-$ $A: D u=w$ where $u \in V^{\frac{1}{2}}(\Omega)$ satisfies

$$
\lambda_{0} w-A(x, \partial) w=0, \nabla \cdot w=0 \text { in } \Omega, w=u \text { on } \Gamma_{i} \text { and } w=0 \text { on } \Gamma_{e} \text {. }
$$

Thus, for $z \in L^{12 / 5}\left(\Omega ; \mathbf{R}^{3}\right)$ we define $b(z, z) \in L^{2}\left(0, T ; \mathcal{D}\left(A^{*}\right)^{\prime}\right)$ by

$$
\langle b(z, z) \mid v\rangle=\int_{\Omega}(\nabla v) z \cdot z, \quad \forall v \in \mathcal{D}\left(A^{*}\right) .
$$

Finally, we define the unbounded operator $\left(\mathcal{D}\left(A_{b}\right), A_{b}\right)=\left(V^{2}\left(\Gamma_{i}\right), P_{b} \Delta_{b}\right)$ in $V^{0}\left(\Gamma_{i}\right)$, where $P_{b} \in \mathcal{L}\left(L^{2}\left(\Gamma_{i}, \mathbf{R}^{3}\right), V^{0}\left(\Gamma_{i}\right)\right)$ denotes the orthogonal projector from $L^{2}\left(\Gamma_{i} ; \mathbf{R}^{3}\right)$ into $V^{0}\left(\Gamma_{i}\right)$.

DEFINITION 1 We shall saythat $(z, u) \in L^{2}\left(0, T ; V^{0}(\Omega)\right) \times L^{2}\left(0, T ; V^{-\frac{1}{2}}\left(\Gamma_{i}\right)\right)$ if $\kappa=0$, or $(z, u) \in L^{2}\left(0, T ; V^{0}(\Omega) \cap L^{12 / 5}\left(\Omega, \mathbf{R}^{3}\right)\right) \times L^{2}\left(0, T ; V^{-\frac{1}{2}}\left(\Gamma_{i}\right)\right)$ if $\kappa=1$, is a weak solution to (6)-(7)-(8) if and only if it obeys the system:

$$
\begin{aligned}
(P z)^{\prime} & =A P z+\left(\lambda_{0}-A\right) P D u+\kappa b(z, z) \in L^{2}\left(0, T ; \mathcal{D}\left(A^{*}\right)^{\prime}\right) \\
u^{\prime} & =A_{b} u+g \in L^{2}\left(0, T ; \mathcal{D}\left(A_{b}\right)^{\prime}\right) \\
P z(0) & =P z_{0} \in V_{n}^{0}(\Omega), u(0)=u_{0} \in V^{-\frac{1}{2}}\left(\Gamma_{i}\right) \\
(I-P) z & =(I-P) D u \in L^{2}\left(0, T ; V^{0}(\Omega)\right), \quad u_{0} \cdot n=z_{0} \cdot n
\end{aligned}
$$

TheOREM 2 Let $(z, p, u, \sigma)$ be an element of $W\left(0, T ; V^{1}(\Omega), V^{-1}(\Omega)\right) \times$ $L^{2}\left(0, T ; L^{2}(\Omega) / \mathbf{R}\right) \times W\left(0, T ; V^{\frac{1}{2}}\left(\Gamma_{i}\right), V^{-\frac{3}{2}}\left(\Gamma_{i}\right)\right) \times L^{2}(0, T)$. Then $(z, p, u, \sigma)$ satisfies (6)-(7)-(8) if and only if $(z, u)$ satisfy (9)-(10)-(11)-(12).

According to [6], the right hand side of (12) is equivalent to $(I-P) z_{0}=$ $(I-P) D u_{0}$. Then (12) ensures that the couple $\left(z, z_{0}\right)$ is entirely determined by its projected part $\left(P z, P z_{0}\right)$ and the boundary values $\left(u, u_{0}\right)$. In the following we only consider the new 'extended' state $Y=(P z, u)$ and the initial condition $Y_{0}=\left(P z_{0}, u_{0}\right)$. We define $\mathcal{H}^{0}=V_{n}^{0}(\Omega) \times V^{-\frac{1}{2}}\left(\Gamma_{i}\right)$ and an adequate unbounded operator $(\mathcal{D}(\mathcal{A}), \mathcal{A})$ in $\mathcal{H}^{0}-\mathcal{A}$ is defined by (19), (20) and studied in section 4 . We introduce the bilinear operator $B$

$$
B\left(Y_{1}, Y_{2}\right)=\left(\begin{array}{c}
b\left(y_{1}+(I-P) D u_{1}, y_{2}+(I-P) D u_{2}\right. \\
0
\end{array}\right) .
$$

Theorem $3 \operatorname{Let}(z, p, u, \sigma) \in W\left(0, T ; V^{1}(\Omega), V^{-1}(\Omega)\right) \times L^{2}\left(0, T ; L^{2}(\Omega) / \mathbf{R}\right)$ $\times W\left(0, T ; V^{\frac{1}{2}}\left(\Gamma_{i}\right), V^{-\frac{3}{2}}\left(\Gamma_{i}\right)\right) \times L^{2}(0, T)$. Then $(z, p, u, \sigma)$ satisfies $(6)-(7)-(8)$ if and only if (12) holds true and the state $Y=(P z, u)$ satisfies

$$
Y^{\prime}=\mathcal{A} Y+\kappa B(Y, Y)+\left(\begin{array}{l}
0 \\
g
\end{array}\right) \in L^{2}\left(0, T ; \mathcal{D}\left(\mathcal{A}^{*}\right)^{\prime}\right), \quad Y(0)=Y_{0} \in \mathcal{H}^{0} .
$$




\subsection{The extended system and the linear quadratic control problem}

The feedback control law is obtained by studying the control problem

$$
(\mathcal{Q})_{z_{0}, u_{0}}^{\infty} \quad \inf \left\{\mathcal{I}(g) \mid g \in L^{2}\left(0, \infty ; V^{0}\left(\Gamma_{i}\right)\right)\right\}
$$

where

$$
\mathcal{I}(g)=\frac{1}{2} \int_{0}^{\infty} \int_{\Omega}\left|\nabla z_{g}\right|^{2}+\frac{1}{2} \int_{0}^{\infty} \int_{\Gamma_{i}}|g|^{2},
$$

and $\left(z_{g}, u_{g}\right) \in W\left(0, \infty ; V^{1}(\Omega) \times V^{\frac{1}{2}}\left(\Gamma_{i}\right), V^{-1}(\Omega) \times V^{-\frac{3}{2}}\left(\Gamma_{i}\right)\right)$ satisfies (6)(7)-(8). If we introduce $\mathcal{C} Y=\nabla(y+(I-P) D u) \in \mathcal{Z}$ with $\mathcal{Z}=L^{2}\left(\Omega ; \mathbf{R}^{9}\right)$ - $\mathcal{C}$ is studied in section 4 lemma 9 - we can rewrite $(\mathcal{Q})_{z_{0}, u_{0}}^{\infty}$ in the form:

$$
\left(\mathcal{P}_{Y_{0}}^{\infty}\right) \quad \inf \left\{\mathcal{J}(g) \mid g \in L^{2}\left(0, \infty ; V^{0}\left(\Gamma_{i}\right)\right)\right\}
$$

where

$$
\mathcal{J}(g)=\frac{1}{2} \int_{0}^{\infty}\left\|\mathcal{C} Y_{g}\right\|_{Z}^{2}+\frac{1}{2} \int_{0}^{\infty} \int_{\Gamma_{i}}|g|^{2},
$$

and $Y_{g}$ satisfies (14) for $\kappa=0$.

\section{Main result}

THEOREM 4 Let $\Pi_{2}$ and $\Pi_{3}$ be the operator defined in (35). Consider the following coupled system,

$$
\begin{aligned}
& \partial_{t} z-\Delta z+(z \cdot \nabla) z_{e}+\left(z_{e} \cdot \nabla\right) z+(z \cdot \nabla) z+\nabla p=0 \text { in } Q \\
& \nabla \cdot z=0 \text { in } Q, \quad z=u \quad \text { in } \Sigma, \quad z=0 \quad \text { in } \Sigma_{e} \\
& \partial_{t} u-\Delta_{b} u+\Pi_{3} u-\sigma n=-\Pi_{2} P z \text { in } \Sigma \\
& z(0)=z_{0} \in V^{\frac{1}{2}}(\Omega), \quad u(0)=u_{0} \in V^{0}\left(\Gamma_{i}\right) .
\end{aligned}
$$

There exists $c_{0}>0$ and $\mu_{0}>0$ such that, if $\delta \in\left(0, \mu_{0}\right)$ and

$$
\begin{gathered}
\left(z_{0}, u_{0}\right) \in \mathcal{W}_{\delta}=\left\{\left(z_{0}, u_{0}\right) \in V^{\frac{1}{2}}(\Omega) \times V^{0}\left(\Gamma_{i}\right) \mid z_{0}-D u_{0} \in V_{0}^{\frac{1}{2}}(\Omega),\right. \\
\left.\left\|u_{0}\right\|_{V^{0}\left(\Gamma_{i}\right)}+\left\|P z_{0}\right\|_{V^{\frac{1}{2}}(\Omega)} \leq c_{0} \delta\right\}
\end{gathered}
$$

then, (15)-(16)-(17)-(18) admit a unique solution in the set

$$
\begin{aligned}
& \mathcal{D}_{\delta}=\{(z, p, u, \sigma) \in W\left(0,+\infty ; V^{\frac{3}{2}}(\Omega), V^{-\frac{1}{2}}(\Omega)\right) \times L^{2}\left(0, \infty ; H^{\frac{1}{2}}(\Omega) / \mathbf{R}\right) \\
& \times W\left(0,+\infty ; V^{1}\left(\Gamma_{i}\right), V^{-1}\left(\Gamma_{i}\right)\right) \times L^{2}(0, \infty) \\
&\|z\|_{L^{2}\left(0,+\infty ; V^{\frac{3}{2}}(\Omega)\right)}+\|u\|_{L^{2}\left(0,+\infty ; V^{1}\left(\Gamma_{i}\right)\right)}+\|\sigma\|_{L^{2}(0, \infty)} \leq \delta \\
&\left.\|p\|_{L^{2}\left(0,+\infty ; H^{\frac{1}{2}}(\Omega)\right)} \leq \delta(1+\delta)\right\} .
\end{aligned}
$$


Moreover, $(z, u)$ obeys

$$
\|z(t)\|_{V^{\frac{1}{2}(\Omega)}}+\|u(t)\|_{V^{0}\left(\Gamma_{i}\right)} \leq C\left(\left\|u_{0}\right\|_{V^{0}\left(\Gamma_{i}\right)}+\left\|P z_{0}\right\|_{V^{\frac{1}{2}}(\Omega)}\right) e^{-\eta t}, \quad t \geq 0 .
$$

\section{The operator $\mathcal{A}$}

The goals of this section are:

- to give a definition of the unbounded operator $(\mathcal{D}(\mathcal{A}), \mathcal{A})$ in $\mathcal{H}^{0}$.

- to characterize the function spaces for which the mapping $Y \mapsto\left(Y^{\prime}-\mathcal{A} Y, Y(0)\right)$ is an isomorphism, in order to have optimal regularity results for the extended system (14) when $\kappa=0$.

- to characterize the functional spaces for which the mapping $Q \mapsto\left(-Q^{\prime}-\mathcal{A}^{*} Q, Q(T)\right)$ is an isomorphism, in order to study the backward adjoint equation which appears in the characterization of the solution to $\left(\mathcal{P}_{Y_{0}}^{\infty}\right)$ - see part 4 .

THEOREM 5

We define the unbounded operator $(\mathcal{D}(\mathcal{A}), \mathcal{A})$ in $\mathcal{H}^{0}=V_{n}^{0}(\Omega) \times V^{-\frac{1}{2}}\left(\Gamma_{i}\right)$ by

$$
\begin{aligned}
\mathcal{D}(\mathcal{A}) & =\left\{(y, u) \in V_{n}^{2}(\Omega) \times V^{\frac{3}{2}}\left(\Gamma_{i}\right) \mid(y-P D u) \in V_{0}^{2}(\Omega)\right\}, \\
\mathcal{A} & =\left(\begin{array}{cc}
A(x, \partial) & \left(\lambda_{0}-A(x, \partial)\right) P D \\
0 & P_{b} \Delta_{b}
\end{array}\right) .
\end{aligned}
$$

The domain $\mathcal{D}(\mathcal{A})$ is dense in $\mathcal{H}^{0}$, and $\mathcal{A}$ generates an analytic semigroup in $\mathcal{H}^{0}$. Moreover, for $0 \leq \theta \leq 1$, the identifications below hold

$$
\begin{aligned}
\mathcal{D}\left(\left(\lambda_{0}-\mathcal{A}\right)^{\theta}\right) & =\left[\mathcal{D}(\mathcal{A}), \mathcal{H}^{0}\right]_{1-\theta}, \\
& =\left\{(y, u) \in V_{n}^{2 \theta}(\Omega) \times V^{2 \theta-\frac{1}{2}}\left(\Gamma_{i}\right) \mid(y-P D u) \in V_{0}^{2 \theta}(\Omega)\right\} .
\end{aligned}
$$

The unbounded operator $\left(\mathcal{D}\left(\mathcal{A}^{*}\right), \mathcal{A}^{*}\right)$ in $\mathcal{H}_{*}^{0}=V_{n}^{0}(\Omega) \times V^{\frac{1}{2}}\left(\Gamma_{i}\right)$ is defined by

$$
\begin{aligned}
\mathcal{D}\left(\mathcal{A}^{*}\right) & =V_{0}^{2}(\Omega) \times V^{\frac{5}{2}}\left(\Gamma_{i}\right), \\
\mathcal{A}^{*} & =\left(\begin{array}{cc}
A^{*}(x, \partial) & 0 \\
D^{*}\left(\lambda-A^{*}(x, \partial)\right) & P_{b} \Delta_{b}
\end{array}\right) .
\end{aligned}
$$

It is the adjoint of $(\mathcal{D}(\mathcal{A}), \mathcal{A})$ with respect to the pivot space $V_{n}^{0}(\Omega) \times V^{0}\left(\Gamma_{i}\right)$. The domain $\mathcal{D}\left(\mathcal{A}^{*}\right)$ is dense in $\mathcal{H}_{*}^{0}$ and $\mathcal{A}^{*}$ generates an analytic semigroup in $\mathcal{H}_{*}^{0}$. Finally, for $0 \leq \theta \leq 1$, the identifications below hold

$$
\mathcal{D}\left(\left(\lambda_{0}-\mathcal{A}^{*}\right)^{\theta}\right)=\left[\mathcal{D}\left(\mathcal{A}^{*}\right), \mathcal{H}_{*}^{0}\right]_{1-\theta}=V_{0}^{2 \theta}(\Omega) \times V^{\frac{1}{2}+2 \theta}\left(\Gamma_{i}\right) .
$$


Moreover, for $0 \leq \theta \leq 1$, we define the function spaces

$$
\begin{aligned}
& \mathcal{H}^{2 \theta}=\left[\mathcal{D}(\mathcal{A}), \mathcal{H}^{0}\right]_{1-\theta}, \mathcal{H}_{*}^{2 \theta}=\left[\mathcal{D}\left(\mathcal{A}^{*}\right), \mathcal{H}_{*}^{0}\right]_{1-\theta}, \\
& \mathcal{H}^{-2 \theta}=\left(\mathcal{H}_{*}^{2 \theta}\right)^{\prime}, \mathcal{H}_{*}^{-2 \theta}=\left(\mathcal{H}^{2 \theta}\right)^{\prime} .
\end{aligned}
$$

Then, as a consequence of the analyticity of the semigroups $\left(e^{\mathcal{A} t}\right)_{t \geq 0}$ and $\left(e^{\mathcal{A}^{*} t}\right)_{t \geq 0}$ respectively in $\mathcal{H}^{0}$ and in $\mathcal{H}_{*}^{0}$, we can state a general isomorphism Theorem (see [1], Chap.3, Thm 2.2, p.166):

THEOREM 6 For every $0 \leq \theta \leq 1$, the mappings below are isomorphisms:

$$
\begin{aligned}
W\left(0, T ; \mathcal{H}^{2 \theta}, \mathcal{H}^{2(\theta-1)}\right) & \rightarrow L^{2}\left(0, T ; \mathcal{H}^{2(\theta-1)}\right) \times\left[\mathcal{H}^{2 \theta}, \mathcal{H}^{2(\theta-1)}\right]_{\frac{1}{2}}, \\
Y & \mapsto\left(Y^{\prime}-\mathcal{A} Y, Y(0)\right), \\
W\left(0, T ; \mathcal{H}_{*}^{2 \theta}, \mathcal{H}_{*}^{2(\theta-1)}\right) & \rightarrow L^{2}\left(0, T ; \mathcal{H}_{*}^{2(\theta-1)}\right) \times\left[\mathcal{H}_{*}^{2 \theta}, \mathcal{H}_{*}^{2(\theta-1)}\right]_{\frac{1}{2}}, \\
Q & \mapsto\left(-Q^{\prime}-\mathcal{A}^{*} Q, Q(T)\right) .
\end{aligned}
$$

Next we determine the spaces $\left[\mathcal{H}^{2 \theta}, \mathcal{H}^{2(\theta-1)}\right]_{\frac{1}{2}}$ of initial conditions.

LEMMA 7 For all $0 \leq \theta \leq 1$ the following characterization holds:

$$
\left[\mathcal{H}^{2 \theta}, \mathcal{H}^{2(\theta-1)}\right]_{\frac{1}{2}}=\mathcal{H}^{2 \theta-1}
$$

Finally, a direct application of Theorem 6 with (23) ensures the existence of a unique solution $Y$ to the extended linear system (14) when $\kappa=0$.

THEOREM 8 Let $g \in L^{2}\left(0, T ; V^{0}\left(\Gamma_{i}\right)\right)$ and $Y_{0} \in \mathcal{H}^{0}$. There exists a unique solution $Y \in L^{2}\left(0, T ; \mathcal{H}^{0}\right)$ to the extended system

$$
Y^{\prime}=\mathcal{A} Y+\left(\begin{array}{l}
0 \\
g
\end{array}\right) \in L^{2}\left(0, T ; \mathcal{D}\left(\mathcal{A}^{*}\right)^{\prime}\right), \quad Y(0)=Y_{0} \in \mathcal{H}^{0}
$$

Moreover, $Y$ belongs to $W\left(0, T ; \mathcal{H}^{1}, \mathcal{H}^{-1}\right)$. More generally, if we assume that $Y_{0} \in \mathcal{H}^{2 \theta}, 0 \leq \theta \leq \frac{1}{2}$, then $Y$ belongs to $W\left(0, T ; \mathcal{H}^{2 \theta+1}, \mathcal{H}^{2 \theta-1}\right)$.

We now treat the backward adjoint equation which appears in the characterization of the solution to $\left(\mathcal{P}_{Y_{0}}^{\infty}\right)$.

LEMMA 9 Let us define $\mathcal{C} \in \mathcal{L}\left(\mathcal{H}^{1}, \mathcal{Z}\right)$ with $\mathcal{Z}=L^{2}\left(\Omega, \mathbf{R}^{9}\right)$ by $\mathcal{C}: Y \rightarrow \nabla(y+(I-P) D u)$. Then

$$
\|\mathcal{C} .\|_{\mathcal{Z}} \sim\|\|_{\mathcal{H}^{1}} \quad \text { and } \quad \mathcal{C}^{*} \mathcal{C} \in \mathcal{L}\left(\mathcal{H}^{\theta+1}, \mathcal{H}_{*}^{\theta-1}\right), \quad 0 \leq \theta \leq 1
$$


Then Theorem 6 with (25) leads to the following theorem.

Theorem 10 Let $Y \in L^{2}\left(0, T ; \mathcal{H}^{1}\right)$. There exists a unique solution $Q \in$ $L^{2}\left(0, T ; \mathcal{H}_{*}^{0}\right)$ to the backward equation

$$
-Q^{\prime}=\mathcal{A}^{*} Q+\mathcal{C}^{*} \mathcal{C} Y, \quad Q(T)=0 .
$$

Moreover, $Q$ belongs to $W\left(0, T ; \mathcal{H}_{*}^{1}, \mathcal{H}_{*}^{-1}\right)$. More generally, if we assume that $Y \in L^{2}\left(0, T ; \mathcal{H}^{2 \theta}\right), \frac{1}{2} \leq \theta \leq 1$, then $Q$ belongs to $W\left(0, T ; \mathcal{H}_{*}^{2 \theta}, \mathcal{H}_{*}^{2(\theta-1)}\right)$.

\section{Resolution of the optimal control problem}

\subsection{The finite time horizon case}

Let $0<T<\infty$ be a finite time horizon. To deal with the optimal control problem $\left(\mathcal{P}_{Y_{0}}^{T}\right)$ we first study the following problem:

$$
\left(\mathcal{P}_{\xi}^{T}\right) \quad \inf \left\{\mathcal{J}_{T}(g) \mid g \in L^{2}\left(0, T ; V^{0}\left(\Gamma_{i}\right)\right)\right\},
$$

where

$$
\mathcal{J}_{T}(g)=\frac{1}{2} \int_{0}^{T}\left\|\mathcal{C} Y_{g}\right\|_{\mathcal{Z}}^{2}+\frac{1}{2} \int_{0}^{T} \int_{\Gamma_{i}}|g|^{2},
$$

and $Y_{g} \in W\left(0, T ; \mathcal{H}, \mathcal{H}^{-1}\right)$ is the solution to

$$
Y^{\prime}=\mathcal{A} Y+\left(\begin{array}{l}
0 \\
g
\end{array}\right), \quad Y(0)=\xi \in \mathcal{H}^{0} .
$$

We introduce the projection operator $\Lambda:(f, g) \in \mathcal{H}_{*}^{0} \mapsto(0, g)$. The problem $\left(\mathcal{P}_{\xi}^{T}\right)$ admits a unique solution $\left(0, g_{\xi, T}\right)$ where $\left(0, g_{\xi, T}\right)=-\Lambda Q_{\xi, T}$ and $\left(Y_{\xi, T}, Q_{\xi, T}\right)$ is the unique solution to the system

$$
\left(S^{T}\right) \quad\left\{\begin{aligned}
Y^{\prime} & =\mathcal{A} Y-\Lambda Q, & & Y(0)=\xi \in \mathcal{H}^{0}, \\
-Q^{\prime} & =\mathcal{A}^{*} Q+\mathcal{C}^{*} \mathcal{C} Y, & & Q(T)=0 .
\end{aligned}\right.
$$

Finally, we denote by $\Pi(T) \in \mathcal{L}\left(\mathcal{H}^{0}, \mathcal{H}_{*}^{0}\right)$, the mapping

$$
\Pi(T): \xi \mapsto Q_{\xi, T}(0) .
$$

\subsection{The infinite time horizon case}

Since, for every $\xi$ and $0<T<\infty$, the solution to $\left(\mathcal{P}_{\xi}^{T}\right)$ has been characterized, we are in position to study the optimal control problem $\left(\mathcal{P}_{\xi}^{\infty}\right)$ and the regularity of its solution in function of the regularity of $\xi$. The problem $\left(\mathcal{P}_{\xi}^{\infty}\right)$ is defined by

$$
\left(\mathcal{P}_{\xi}^{\infty}\right) \quad \inf \left\{\mathcal{J}(g) \mid g \in L^{2}\left(0, \infty ; V^{0}\left(\Gamma_{i}\right)\right)\right\}
$$


where the following functional satisfies (28):

$$
\mathcal{J}(g)=\frac{1}{2} \int_{0}^{\infty}\left\|\mathcal{C} Y_{g}\right\|_{\mathcal{Z}}^{2}+\frac{1}{2} \int_{0}^{\infty} \int_{\Gamma_{i}}|g|^{2}, \quad Y_{g} \in W\left(0, \infty ; \mathcal{H}, \mathcal{H}^{-1}\right) .
$$

Using a null controllability result stated in [3] we can show that there exists a control $g \in L^{2}\left(0, \infty ; V^{0}\left(\Gamma_{i}\right)\right)$ such that $\mathcal{J}(g)<+\infty$. This gives us the existence of a unique solution $g_{\xi}$ to $\left(\mathcal{P}_{\xi}^{\infty}\right)$.

THEOREM 11 The problem $\left(\mathcal{P}_{\xi}^{\infty}\right)$ admits a unique solution $g_{\xi}$ where $g_{\xi}=-\psi_{\xi}$ and $\left(Y_{\xi}, Q_{\xi}\right)=\left(\left(y_{\xi}, u_{\xi}\right),\left(\Phi_{\xi}, \psi_{\xi}\right)\right) \in W\left(0, \infty ; \mathcal{H}^{1}, \mathcal{H}^{-1}\right) \times$ $W\left(0, \infty ; \mathcal{H}_{*}^{1}, \mathcal{H}_{*}^{-1}\right)$ is the unique solution to the system:

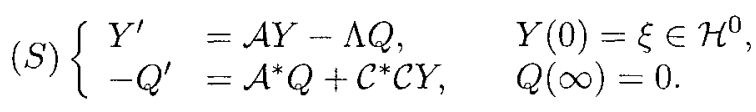

Moreover, there exists $\Pi \in \mathcal{L}\left(\mathcal{H}^{*}, \mathcal{H}_{*}^{0}\right)$, $\Pi^{*}=\Pi$ such that

$$
Q_{\xi}=\Pi Y_{\xi}, \quad \mathcal{J}\left(g_{\xi}\right)=\frac{1}{2}\langle\Pi \xi \mid \xi\rangle_{\mathcal{H}_{*}^{0}, \mathcal{H}^{0}}
$$

The control $g_{\xi}$ has been calculated as the limit of $g_{\xi, T}$ when $T \rightarrow \infty$, where $g_{\xi, T}$ is the unique solution to $\left(\mathcal{P}_{\xi}^{T}\right)$.

We now focus on the properties of $\Pi$. First, from the limit $\Pi(T) \rightarrow \Pi$ as $T \rightarrow \infty$ we can show that $\Pi$ satisfies an algebraic Riccati equation. Next, Theorems 8 and 10 for $(S)$ lead to sharp regularity result for $\Pi$.

THEOREM 12 II satisfies a Riccati equation: $\forall(\xi, \zeta) \in \mathcal{H}^{1} \times \mathcal{H}^{1}$,

$$
\langle\Pi \xi \mid \mathcal{A} \zeta\rangle_{\mathcal{H}_{*}^{1}, \mathcal{H}^{-1}}+\langle\mathcal{A} \xi \mid \Pi \zeta\rangle_{\mathcal{H}^{-1}, \mathcal{H}_{*}^{1}}+(\mathcal{C} \xi \mid \mathcal{C} \zeta)_{\mathcal{Z}}-(\Lambda \Pi \xi \mid \Lambda \Pi \zeta)_{V^{0}\left(\Gamma_{i}\right)}=0
$$

and the regularizing property $\quad \Pi \in \mathcal{L}\left(\mathcal{H}^{2 \theta}, \mathcal{H}_{*}^{2 \theta}\right), \quad 0 \leq \theta \leq \frac{1}{2}$.

Next we set $\mathcal{A}_{\Pi}=(\Lambda \Pi-\mathcal{A})$, so that the optimal trajectory $Y_{\xi}$ satisfy

$$
Y^{\prime}+\mathcal{A}_{\Pi} Y=0, \quad Y(0)=\xi \in \mathcal{H}^{0}
$$

From a classical result due to Datko in [5, Chap 4, Thm 4.1], we show the exponential stability of $e^{-\mathcal{A}_{\Pi} t} Y_{0}$. Let $\alpha>0$, the positivity of $\mathcal{A}_{\Pi}$ allows us to define its fractional power $\mathcal{A}_{\Pi}^{\alpha}$ and we respectively identify $\mathcal{D}\left(\mathcal{A}_{\Pi}^{\alpha}\right)$ and $\mathcal{D}\left(\mathcal{A}_{\Pi}^{* \alpha}\right)$ with $\mathcal{D}\left(\left(\lambda_{0}-\mathcal{A}\right)^{\alpha}\right)$ and $\mathcal{D}\left(\left(\lambda_{0}-\mathcal{A}^{*}\right)^{\alpha}\right)$. Thus (21) and (22) ensures a characterization $\mathcal{D}\left(\mathcal{A}_{\Pi}^{\alpha}\right)$ and $\mathcal{D}\left(\mathcal{A}_{\Pi \Pi}^{* \alpha}\right)$.

THEOREM 13 The unbounded operator $\left(\mathcal{D}\left(-\mathcal{A}_{\Pi}\right),-\mathcal{A}_{\Pi}\right)$ generates an analytic exponentially stable semigroup in $\mathcal{H}^{0}$ and the characterizations below hold

$$
\mathcal{D}\left(\mathcal{A}_{\Pi}^{\theta}\right)=\mathcal{H}^{2 \theta}, \quad \mathcal{D}\left(\mathcal{A}_{\Pi}^{* \theta}\right)=\mathcal{H}_{*}^{2 \theta}, \quad 0 \leq \theta \leq 1
$$


Next we define new norms in the spaces $\mathcal{H}^{\alpha}$ and $\mathcal{H}^{1+\alpha}$ which are essential to study the stabilization of the Navier-Stokes equation (see section $\mathbf{6}$ ).

Definition 14 We define $\Pi{ }^{(\alpha)} \in \mathcal{L}\left(\mathcal{H}^{\alpha}, \mathcal{H}_{*}^{-\alpha}\right)$ by $\Pi^{(\alpha)}=\mathcal{A}_{\Pi}{ }^{* \frac{\alpha}{2}} \Pi \mathcal{A}_{\Pi}{ }^{\frac{\alpha}{2}}$.

THEOREM 15 The operator $\Pi^{(\alpha)}$ has the regularizing property:

$$
\Pi^{(\alpha)} \in \mathcal{L}\left(\mathcal{H}^{2 \theta+\alpha}, \mathcal{H}_{*}^{2 \theta-\alpha}\right), \quad 0 \leq \theta \leq \frac{1}{2}
$$

Definition 16 We define the two mappings $\mathcal{N}_{\alpha}$ and $\mathcal{R}_{1+\alpha}$ by

$$
\begin{aligned}
\mathcal{N}_{\alpha}(\xi) & =\left(\left\langle\Pi^{(\alpha)} \xi \mid \xi\right\rangle_{\mathcal{H}_{*}^{-\alpha}, \mathcal{H}^{\alpha}}\right)^{\frac{1}{2}}, \quad \xi \in \mathcal{H}^{\alpha} \\
\mathcal{R}_{1+\alpha}(\xi) & =\left(\left\langle\mathcal{A}_{\Pi} \xi \mid \Pi^{(\alpha)} \xi\right\rangle_{\mathcal{H}^{-1+\alpha}, \mathcal{H}_{*}^{1-\alpha}}\right)^{\frac{1}{2}}, \quad \xi \in \mathcal{H}^{1+\alpha} .
\end{aligned}
$$

THEOREM $17 \mathcal{N}_{\alpha}$ and $\mathcal{R}_{1+\alpha}$ define norms respectively on $\mathcal{H}^{\alpha}$ and $\mathcal{H}^{1+\alpha}$,

$$
\mathcal{N}_{\alpha}(\cdot) \sim\|\cdot\|_{\mathcal{H}^{\alpha}}, \quad \mathcal{R}_{1+\alpha}(\cdot) \sim\|\cdot\|_{\mathcal{H}^{1+\alpha}}
$$

We shall point out that the expression of $\mathcal{R}_{1+\alpha}(\xi)$ is explicitly given by $\left\langle\mathcal{A}_{\Pi} \xi \mid \Pi^{(\alpha)} \xi\right\rangle_{\mathcal{H}^{-1+\alpha}, \mathcal{H}_{*}^{1-\alpha}}=\frac{1}{2}\left\|\mathcal{C} \mathcal{A}_{\Pi} \frac{\alpha}{2} \xi\right\|_{\mathcal{Z}}^{2}+\frac{1}{2}\left\|\Lambda \Pi \mathcal{A}_{\Pi} \frac{\alpha}{2} \xi\right\|_{V^{0}\left(\Gamma_{i}\right)}^{2}, \quad \forall \xi \in \mathcal{H}^{1+\alpha}$

which follows from (31) in which we have replaced $\xi$ and $\zeta$ by $\mathcal{A}_{\Pi}^{\frac{\alpha}{2}} \xi$.

We finish this section by giving the PDE formulation of the closed loop system (32).

THEOREM 18 The operator $\Pi$ can be rewritten as follows,

$$
\Pi=\left(\begin{array}{ll}
\Pi_{1} & \Pi_{2}^{*} \\
\Pi_{2} & \Pi_{3}
\end{array}\right), \quad \begin{aligned}
& \Pi_{1} \in \mathcal{L}\left(V_{n}^{0}(\Omega)\right), \\
&
\end{aligned}
$$

where $\Pi_{1}$ and $\Pi_{3}$ are positive, definite and self-adjoint operators. Then $Y=$ $(P z, u) \in W\left(0, \infty ; \mathcal{H}^{1}, \mathcal{H}^{-1}\right)$ satisfies (32) if and only if the element $(z, p, u, \sigma)$ of $W\left(0, \infty ; V^{1}(\Omega), V^{-1}(\Omega)\right) \times L^{2}\left(0, \infty ; L^{2}(\Omega) / \mathbf{R}\right) \times W\left(0, \infty ; V^{\frac{1}{2}}\left(\Gamma_{i}\right), V^{-\frac{3}{2}}\left(\Gamma_{i}\right)\right)$ $\times L^{2}(0, \infty)$ satisfies

$$
\begin{aligned}
& \partial_{t} z-\Delta z+(z \cdot \nabla) z_{e}+\left(z_{e} \cdot \nabla\right) z+\nabla p=0, \quad \nabla \cdot z=0 \text { in } Q \\
& \partial_{t} u-\Delta_{b} u+\Pi_{3} u-\sigma n=-\Pi_{2} P z, \quad z=u \text { in } \Sigma_{i}, \quad z=0 \text { in } \Sigma_{e} \\
& z(0)=z_{0} \in V^{0}(\Omega), \quad u(0)=u_{0} \in V^{-\frac{1}{2}}\left(\Gamma_{i}\right), \quad z_{0} \cdot n=u_{0} \cdot n
\end{aligned}
$$




\section{Stabilization of the Navier-Stokes equations}

We now come back to the stabilization of the Navier-Stokes system. We now consider the nonlinear system (15)-(16)-(17)-(18) which we can rewrite in the abstract formulation

$$
Y^{\prime}+\mathcal{A}_{\Pi} Y=B(Y, Y), \quad Y(0)=Y_{0} \in \mathcal{H}^{\frac{1}{2}}
$$

Finally, Theorem 4 is a direct consequence of the following result.

THEOREM 19 There exists $c_{0}>0$ and $\mu_{0}>0$ such that, if $\delta \in\left(0, \mu_{0}\right)$ and $Y_{0} \in \mathcal{V}_{\delta}=\left\{Y \in \mathcal{H}^{\frac{1}{2}} \mid \quad\|Y\|_{\mathcal{H}^{\frac{1}{2}}}<c_{0} \delta\right\}$ then, (36) admit a unique solution in the set $\mathcal{S}_{\delta}=\left\{Y \in W\left(0, \infty ; \mathcal{H}^{\frac{3}{2}}, \mathcal{H}^{-\frac{1}{2}}\right) \mid\|Y\|_{W\left(0, \infty ; \mathcal{H}^{\frac{3}{2}}, \mathcal{H}^{-\frac{1}{2}}\right)} \leq \delta\right\}$.

Moreover, there exists $\eta>0$ such that

$$
\|Y(t)\|_{\mathcal{H}^{\frac{1}{2}}} \leq C\left\|Y_{0}\right\|_{\mathcal{H}^{\frac{1}{2}}} e^{-\eta t}
$$

Proof. Here, we give a brief sketch of the proof of the stability result. We multiply the left hand side of (36) by $\Pi^{\left(\frac{1}{2}\right)} Y(t)$. According to (34) with $\alpha=\frac{1}{2}$ we obtain

$$
\frac{1}{2} \frac{d}{d t} \mathcal{N}_{\frac{1}{2}}^{2}(Y(t))+\mathcal{R}_{\frac{3}{2}}^{2}(Y(t))=\left\langle B(Y(t), Y(t)) \mid \Pi^{\left(\frac{1}{2}\right)} Y(t)\right\rangle
$$

Then we invoke a classical estimation - see [2, Chap.6, 6.9 and 6.10] - to obtain $\left|\left\langle B(Y(t), Y(t)) \mid \Pi^{\left(\frac{1}{2}\right)} Y(t)\right\rangle\right| \leq C\|Y(t)\|_{\mathcal{H}^{\frac{1}{2}}}\|Y(t)\|_{\mathcal{H}^{\frac{1}{2}}}\left\|\Pi^{\left(\frac{1}{2}\right)} Y(t)\right\| \|_{\mathcal{H}^{\frac{1}{2}}}$. According to (33) with $\alpha=\theta=\frac{1}{2}$ it yields

$$
\left|\left\langle B(Y(t), Y(t)) \mid \Pi^{\left(\frac{1}{2}\right)} Y(t)\right\rangle\right| \leq C\|Y(t)\|_{\mathcal{H}^{\frac{1}{2}}}\|Y(t)\|_{\mathcal{H}^{\frac{3}{2}}}^{2} .
$$

Thus (34) gives us $C_{0}>0$ such that

$$
\frac{d}{d t} \mathcal{N}_{\frac{1}{2}}^{2}(Y(t))+2\left(1-C_{0} \mathcal{N}_{\frac{1}{2}}(Y(t))\right) \mathcal{R}_{\frac{3}{2}}^{2}(Y(t) \leq 0
$$

It is obvious to see that if $\mathcal{N}_{\frac{1}{2}}\left(Y_{0}\right)$ is small enough, we can choose $\mathcal{N}_{\frac{1}{2}}\left(Y_{0}\right)<$ $\frac{1}{4 C_{0}}$, so that the mapping $t \rightarrow \mathcal{N}_{\frac{1}{2}}(Y(t))$ be a nonincreasing function with values less than $\frac{1}{4 C_{0}}$. This gives the existence of $\delta$, and (37) follows from (38).

\section{References}

[1] A. Bensoussan, G. Da Prato, M.C. Delfour, S.K. Mitter Representation and Control of Infinite Dimentional Systems, Volume I. Birkhauser, Boston, 1992. 
[2] P. Constantin, C. Foias. Navier-Stokes Equations. University of Chicago Press, Chicago Lectures in Mathematics, 1988.

[3] E. Fernández-Cara, S. Guerrero, O. Yu. Imanuvilov, J.-P. Puel. Local exact controllability of the Navier-Stokes system. to appear in J. Math. Pures et Appliquées. .

[4] G.P. Galdi. An Introduction to the Mathematical Theory of the Navier-Stokes Equations, Vol. II., Nonlinear Steady Problems. Springer, New York, 1994.

[5] A. Pazy. Semigroups of Linear Operators and Applications to Partial Differential Equations. Springer-Verlag, New York, 1983.

[6] J.-P. Raymond. Stokes and Navier-Stokes Equations with Nonhomogeneous Boundary Conditions, preprint, 2005. 